\title{
Application of the Screened Hydrogenic Model to Light Atoms
}

\author{
Robert W. Smithwick \\ Analytical Chemistry Organization, Y-12 National Security Complex, Oak Ridge, USA \\ Email: Robert.Smithwickiii@cns.doe.gov
}

How to cite this paper: Smithwick, R.W. (2021) Application of the Screened Hydrogenic Model to Light Atoms. Computational Chemistry, 9, 131-143.

https://doi.org/10.4236/cc.2021.93008

Received: April 11, 2021

Accepted: May 31, 2021

Published: June 3, 2021

Copyright $\odot 2021$ by author(s) and Scientific Research Publishing Inc. This work is licensed under the Creative Commons Attribution International License (CC BY 4.0).

http://creativecommons.org/licenses/by/4.0/

\begin{abstract}
The purpose of this work was to develop a Screened Hydrogenic Model (SHM) to accurately calculate the electron energies for light atoms and ions with up to ten electrons for atomic numbers up to 18. The total energy of an atom or ion was calculated with effective nuclear charges and screening parameters for each electron type ( $1 s, 2 s$ and $2 p$ ) within a specific electron configuration. Multiple energy states, centered at the total energy, were calculated for electron configurations that have Russell-Saunders coupling. The energy of each electron included its relativistic energy, $E_{R E L}$, but close overall agreement between the calculated and experimental energies of multi-electron configurations required that the one-electron expression for $E_{R E L}$ be modified in a simple manner. In the present work, $98 \%$ of the 587 calculated energies for light atoms/ions have a relative error within $\pm 0.1 \%$ of the corresponding experimental energies. The effective nuclear charges described in this work allow hydrogen-like wave functions to be defined for the electrons within a multi-electron configuration. The SHM, described in this work, is available for future calculations involving light atoms and ions.
\end{abstract}

\section{Keywords}

Atom, Electron, Screening, Quantum, Plasma

\section{Introduction}

It is well known that the energy levels of a one-electron atom or ion have been determined by solving the Schrödinger equation. The energy of a multi-electron atom or ion is significantly more difficult to calculate with a modified Schrödinger equation because the electrons repel one another as they orbit the nucleus. One theoretical approach for determining the energy of complex atoms and ions is the self-consistent field (SCF) method. This approach starts with a modified Schrödinger equation, calculates the energy with a basis set of atomic functions, 
modifies the potential-energy term, and iteratively repeats this process by computer while solving for minimum total energy. This process is repeated until the potential energy values for the electrons are the same as the potential energies that were used to calculate them. This approach, which can also be applied to molecular calculations, gives increasingly better agreement as the basis set is expanded and the complexity of the calculation is increased.

The Screened Hydrogenic Model (SHM) is an alternative approach that uses the one-electron wavefunctions of the hydrogen atom for each electron of a multi-electron atom by substituting an effective nuclear charge $Z_{i}$ for the value of $Z$. Agreement with experimental energies can be obtained by varying $Z_{i}$ with screening parameters. The SHM provides a starting point for semi-empirical calculations involving complex atomic or molecular systems.

The SHM was first used by Slater [1] [2] who approximated the energies of electrons in $1 \mathrm{~s}$ and $2 \mathrm{~s} / 2 \mathrm{p}$ orbitals as $E=-Z_{i}^{2} /\left(2 n_{i}^{2}\right)$ in hartree atomic units (a.u.). $Z_{1 S}$ (with $n_{i}=1$ ) equals $Z-0.30$ when two 1 s electrons were present and both $Z_{2 S}$ and $Z_{2 P}$ (with $n_{i}=2$ ) equals $Z-1.70-0.35\left(N_{2 S}+N_{2 P}-1\right)$ when two 1s electrons were present. The Slater 1s wave function was the same as the hydrogen $1 \mathrm{~s}$ wave function with $Z_{1 S}$ substituted for $Z$. The Slater $2 \mathrm{~s}$ and $2 \mathrm{p}$ wave functions both had the same value of $Z_{2 S}=Z_{2 p}$ substituted for $Z$ and the same radial part of the hydrogen-like $2 \mathrm{p}$ wave function but with $2 \mathrm{~s}$ or $2 \mathrm{p}$ angular parts. Numerous molecular orbital calculations were based on Slater wave functions. Layzer [3] [4] [5] and Kregar [6] [7] each calculated screening parameters and electron energies with series functions in powers of $Z$.

The calculation of the properties of dense plasmas at high temperatures [8]-[14] is an important application of the SHM that involves the determination of the energies of atoms and ions across the periodic table. The Dirac equation, which will be discussed in the next section, is an alternative form of the SHM that includes relativistic corrections for each electron. A closer agreement between experimental and calculated electron energies is expected to lead to improved predictions of plasma properties.

In the present work, the SHM will be used for atoms and ions with up to 10 (1s, 2 s and 2p) electrons with $Z$ up to 18 to obtain close agreement between calculated and experimental energies. The effective nuclear charges will be calculated for individual configurations. The effective nuclear charges for $2 \mathrm{~s}$ orbitals will be treated separately from those of $2 p$ orbitals. Values of the screening parameters will be determined by the criterion of the lowest sum-squared error between the calculated energies and the corresponding 587 experimental electron energies of light atoms. Relativistic corrections will be made for each electron with a modified parameter to be described. Hydrogen-like wave functions, which can be written straightforwardly for these electrons, will be available for future calculations.

\section{Equations for the Electron Energies}

The Dirac fine-structure equation (see Bethe [15]: Eqn. 17.1) gives the exact 
binding energy of a single electron in a hydrogen-like atom or ion (in hartree atomic units):

$$
\begin{aligned}
& E \text { (1-electron })=\left(E-E_{o}\right) /\left(\alpha^{2} E_{o}\right) \\
& =\left(1 / \alpha^{2}\right)\left\{\left[1+\left\{\alpha Z /\left[n_{i}-\left|j+\frac{1}{2}\right|+\left(\left(j+\frac{1}{2}\right)^{2}-\alpha^{2} Z^{2}\right)^{\frac{1}{2}}\right]\right\}^{2}\right]^{-\frac{1}{2}}-1\right\}
\end{aligned}
$$

In this equation, $Z$ is the atomic number, $n_{i}$ is the principle quantum number, $\alpha$ is the hyperfine constant, and $j$ is the total angular momentum quantum number equal to $S+L$. The relativistic energy, $E_{o}=m_{e} c^{2}$, of the rest mass $m_{e}$ of the electron has been subtracted. One hartree (atomic unit) equals $\alpha^{2} m_{e} c^{2}$, where $a^{2}=5.3251 \times 10^{-5}$. This equation can be expanded in powers of $(\alpha Z)^{2}$ (see Mizushima [16], Eqn. 7-87) to give the following equation (in a.u.):

$$
E(1 \text {-electron })=-Z^{2} /\left(2 n_{i}^{2}\right)-\left[\alpha^{2} Z^{4} /\left(2 n_{i}^{3}\right)\right]\left[1 /\left(j+\frac{1}{2}\right)-3 /\left(4 n_{i}\right)+\cdots\right]
$$

In Equation (2), the first term $-Z^{2} /\left(2 n_{i}^{2}\right)$ equals the energy of the Schrödinger equation and the second term is the relativistic correction $E_{\mathrm{REL}}$ in atomic units.

The total energy for a multi-electron configuration, $1 \mathrm{~s}^{N_{1 S}} 2 \mathrm{~s}^{N_{2 S}} 2 \mathrm{p}^{N_{2 P}}$, of the Screened Hydrogenic Model (SHM) is the following summation of one-electron energies where $Z_{i}$ is the effective nuclear charge calculated with screening parameters that are specific to the number, $N_{i}$, of electrons in different orbitals (in a.u.):

$$
E_{Z, N_{1 S}, N_{2 S}, N_{2 P}}=-\sum\left[N_{i} Z_{i}^{2} /\left(2 n_{i}^{2}\right)+E_{\mathrm{REL}}\right] .
$$

An alternative to Equation (3) is the summation of the total energy for the electrons with Equation (1) modified with $Z_{i}$ for multi-electron configurations.

Snyder [17] [18] proposed the following equation for $E_{R E L}$ for the electrons of multi-electron configurations, where $Z_{i}$ has been substituted in Equation (2) for $Z$ (in a.u.):

$$
E_{\mathrm{REL}}=-\sum N_{i} \alpha^{2}\left[Z_{i}^{4} /\left(2 n_{i}^{3}\right)\right]\left[1 /\left(j+\frac{1}{2}\right)-3 /\left(4 n_{i}\right)\right] .
$$

In the present work, poor overall results were observed when $j$ was set equal to $S+L$ in Equation (4) to calculate $E_{R E L}$ and $E_{Z, N_{1 S}, N_{2 S}, N_{2 P}}$ in Equation (3) as the sum over multiple electrons in atoms and ions. However, when $\mathrm{j}$ of Equation (4) was empirically set to a constant value of $1 / 2$, the resulting Equation (5) gave good overall agreement between calculated and experimental energies for configurations with $1 \mathrm{~s}, 2 \mathrm{~s}$ and $2 \mathrm{p}$ electrons. The constant value of $j=1 / 2$ is consistent with $E_{\mathrm{REL}}$ being determined by the intrinsic electron spin, $s=1 / 2$, of each electron in the summation rather than by $j=S+L$ of the atomic state(s) of the multi-electron configuration.

$$
E_{\mathrm{REL}}=-\sum N_{i}\left[\left\{\alpha^{2} Z_{i}^{4}\left[1-3 /\left(4 n_{i}\right)\right] /\left(2 n_{i}^{3}\right)\right\}-\Lambda_{i, Z}\right]
$$


The Retherford-Lamb correction, $\Lambda_{i, Z}$, has been added to Equation (5) but only the $1 \mathrm{~s}$ electrons will be included in the present work. When there is one electron in the $1 \mathrm{~s}$ orbital, values of $\Lambda_{1 S, Z}$ were calculated from Kabir ([19]: Eqn. 4); see also Garcia [20]). Values in units of Rydbergs were divided by two to convert them to a.u. When there are two electrons in the 1s orbital, the values of $\Lambda_{1 S, Z}$ (in a.u.) were calculated together for both electrons from Kabir ([19]: Eqn. 5). A value of $\Lambda_{1 S, Z}$ shown in Equation (5) is the correction per 1s electron. Hartmann ([21]: Table V) lists these values for $Z$ from 2 to 36 . These calculations of $\Lambda_{1 S, Z}$ follow the notation of Kabir [19] that uses $Z$ rather than $Z_{i}$.

\section{Parameters for the Screened Hydrogenic Model}

In the following equations, $Z_{i}$ is the effective nuclear charge for orbital " $"$ " and $N_{i}$ is the number of electrons in each orbital type (1s, $2 \mathrm{~s}$ and $2 \mathrm{p})$ of a given configuration. The numbers shown in square brackets and the numerical values of parameters, $C_{1}$ through $C_{9}$, will be described later.

$$
\begin{gathered}
Z_{1 S}=Z-\left[0.3125-0.030 / Z-0.006 / Z^{2}\right]\left(N_{1 S}-1\right)-C_{1} N_{2 S}-C_{2} N_{2 P} \\
Z_{2 S}=Z-C_{3} N_{1 S}-C_{4}\left(N_{2 S}-1\right)-C_{5} N_{2 P} \\
Z_{2 P}=Z-C_{6} N_{1 S}-C_{7} N_{2 S}-\left[C_{8}-C_{9} / Z\right]\left(N_{2 P}-1\right)
\end{gathered}
$$

The $\left(N_{i}-1\right)$ factors for each orbital type are needed to exclude self-screening by a single electron of the same orbital type. For example, the 1 s electron of the hydrogen atom does not screen itself from the nucleus.

In some configurations, the electron energy is split into 2, 3, 4 or 6 states by Russell-Saunders coupling. The notation of Slater ([2]: p. 343), shown in Table 1 for those configurations, is used to calculate the energies of the individual levels centered at $E_{a V}$ that equals $E_{Z, N_{1 S}, N_{2 S}, N_{2 P}}$ of Equation (3). $E_{a V}$ is the weighted-average energy of multiple states of a configuration where the weighting factor for

\begin{tabular}{|c|c|}
\hline $\mathrm{sp}$ or $\mathrm{sp}^{5}$ & $\mathrm{sp}^{2}$ or $\mathrm{sp}^{4}$ \\
\hline $\begin{array}{l}{ }^{3} \mathrm{P}^{\mathrm{o}}: E_{a V}-1 / 6 G^{1}(\mathrm{sp}) \\
{ }^{1} \mathrm{P}^{\mathrm{o}}: E_{a V}+1 / 2 G^{1}(\mathrm{sp})\end{array}$ & $\begin{array}{l}{ }^{4} \mathrm{P}: E_{a v}-3 / 25 F^{2}(\mathrm{pp})-1 / 3 G^{1}(\mathrm{sp}) \\
{ }^{2} \mathrm{P}: E_{a v}-3 / 25 F^{2}(\mathrm{pp})+2 / 3 G^{1}(\mathrm{sp})\end{array}$ \\
\hline $\mathrm{p}^{2}$ or $\mathrm{s}^{2} \mathrm{p}^{2}$ or $\mathrm{p}^{4}$ or $\mathrm{s}^{2} \mathrm{p}^{4}$ & $\begin{array}{l}{ }^{2} \mathrm{D}: E_{a v}+3 / 25 F^{2}(\mathrm{pp}) \\
{ }^{2} \mathrm{~S}: E_{a v}+12 / 25 F^{2}(\mathrm{pp})\end{array}$ \\
\hline \multirow{2}{*}{$\begin{array}{l}{ }^{3} \mathrm{P}: E_{a v}-3 / 25 F^{2}(\mathrm{pp}) \\
{ }^{1} \mathrm{D}: E_{a V}+3 / 25 F^{2}(\mathrm{pp}) \\
{ }^{1} \mathrm{~S}: E_{a v}+12 / 25 F^{2}(\mathrm{pp})\end{array}$} & $\mathrm{sp}^{3}$ \\
\hline & ${ }^{5} S^{0}: E_{a V}-9 / 25 F^{2}(\mathrm{pp})-1 / 2 G^{1}(\mathrm{sp})$ \\
\hline $\mathrm{p}^{3}$ or $\mathrm{s}^{2} \mathrm{p}^{3}$ & $\begin{array}{c}{ }^{3} S^{o}: E_{a v}-9 / 25 F^{2}(\mathrm{pp})+5 / 6 G^{1}(\mathrm{sp}) \\
{ }^{3} \mathrm{D}^{\mathrm{o}}: E_{a v}-1 / 6 G^{1}(\mathrm{sp})\end{array}$ \\
\hline $\begin{array}{l}{ }^{4} \mathrm{~S}^{\mathrm{o}}: E_{a v}-9 / 25 F^{2}(\mathrm{pp}) \\
{ }^{2} \mathrm{D}^{\mathrm{o}}: E_{a v} \\
{ }^{2} \mathrm{P}^{\mathrm{o}}: E_{a v}+6 / 25 F^{2}(\mathrm{pp})\end{array}$ & $\begin{array}{c}{ }^{1} \mathrm{D}^{\mathrm{o}}: E_{a V}+1 / 2 G^{1}(\mathrm{sp}) \\
{ }^{3} \mathrm{P}^{\mathrm{o}}: E_{a v}+6 / 25 F^{2}(\mathrm{pp})-1 / 6 G^{1}(\mathrm{sp}) \\
{ }^{1} \mathrm{P}^{\mathrm{o}}: E_{a v}+6 / 25 F^{2}(\mathrm{pp})+1 / 2 G^{1}(\mathrm{sp})\end{array}$ \\
\hline
\end{tabular}
each state is $(2 S+1)(2 L+1)$. For example, a ${ }^{4} \mathrm{P}$ state has a weighting factor

Table 1. Energies of multiplets of certain configurations with $\mathrm{s}$ and $\mathrm{p}$ electrons from [2]. 
of $4 \times 3$. The calculation of the energies of the states may involve couplings, $G^{1}$ (1s, 2p), $F^{2}(2 \mathrm{p}, 2 \mathrm{p})$, and/or $G^{1}(2 \mathrm{~s}, 2 \mathrm{p})$.

In this work, the $G^{1}$ and $F^{2}$ couplings will be estimated by the following equations (in a.u.). The numerical values of parameters, $C_{10}$ through $C_{15}$, will be described later.

$$
\begin{gathered}
G^{1}(1 \mathrm{~s}, 2 \mathrm{p})=C_{10} Z_{2 P}-C_{11} \\
F^{2}(2 \mathrm{p}, 2 \mathrm{p})= \begin{cases}C_{12} Z_{2 P} & \text { if } N_{2 S}=0 \\
C_{13} Z_{2 P} & \text { if } N_{2 S}=1 \\
C_{14} Z_{2 P} & \text { if } N_{2 S}=2\end{cases} \\
G^{1}(2 \mathrm{~s}, 2 \mathrm{p})=C_{15} Z_{2 P} .
\end{gathered}
$$

\section{Treatment of Experimental Energies}

The energies for each element and configuration $E_{Z, N_{1 S}, N_{2 S}, N_{2 P}}$ are computed by adding the energies from spectroscopic data [22] [23] that are required to remove electrons from the atom or ion, starting with the ionization potential of the 1-electron ion for each element. The calculated values of the energies of the 1 -electron ions were corrected for the reduced mass of the electron. The subsequent ionization potentials and transitions in wave numbers were divided by $-219,474.62 \mathrm{~cm}^{-1}$ per hartree to convert them into a negative energy in a.u. For example, the energy of the $1 s^{2} 2 s^{1}$ ground state of lithium involves the sum of the three ionization potentials of lithium. The energy of the excited state of lithium in the $1 \mathrm{~s}^{2} 2 \mathrm{p}^{1}$ configuration equals the same sum of the three ionization potentials minus the transition energy from the ground state of lithium to its $1 s^{2} 2 p^{1}$ excited state, resulting in a total energy that is less negative than the ground state.

For certain $\mathrm{P}$ and $\mathrm{D}$ states, fine structure due to $j$ coupling in the form of doublets, triplets, etc. has been tabulated in the literature [22] [23] with their assigned values of $j$. In these cases, an average experimental energy, weighted by $(2 j+1)$, has been calculated for use in the present work. The configuration, $1 s^{1} 2 s^{1}$, has ortho and para terms, ${ }^{3} S$ and ${ }^{1} S$ with nearly the same energy. When these terms were listed separately, a three-to-one weighted average was used to calculate the average experimental energy of the configuration.

The experimental energies, computed in this work from spectroscopic data for the neutral atoms of helium through neon, were compared to the corresponding experimental total energies listed by Veillard [24]. The energies of these nine atoms each agreed within \pm 0.001 a.u., except for oxygen that agreed within \pm 0.02 a.u. (compared to its total experimental energy of -75.1101 a.u.).

\section{Computational Methods}

In the present work, 587 energy states in 293 different configurations of atoms and ions of helium through argon have been used to calculate fifteen parameters, $C_{1}$ to $C_{15}$. Configurations with up to a total of ten ( $1 \mathrm{~s}, 2 \mathrm{~s}$ and $2 \mathrm{p}$ ) electrons of 
atoms or ions and up to an atomic number of 18 have been included. The parameters were determined by the criterion of least-squares error by the use of alternating cycles of computation. The algorithm involved a simple search that successively varied parameters $C_{1}$ through $C_{9}$ in step changes ( $-1 \mathrm{step}, 0$ step and +1 step) per parameter and the process was repeated until no further reduction in the calculated sum-squared error was observed for a given step size. The step size was then reduced in half (eventually down to $10^{-4}$ ) to find the values of the parameters that gave the lowest sum-squared error. The number of combinations that were searched was $3^{9}=\sim 20,000$ per cycle. For each cycle, Equation (3) was used to vary and test parameters to find the lowest sum-squared error between calculated and experimental energies.

Parameters $C_{10}$ through $C_{15}$ of Equations (9) through (11) were used to estimate the values of $G^{1}(1 \mathrm{~s}, 2 \mathrm{p}), F^{2}(2 \mathrm{p}, 2 \mathrm{p})$ and $G^{1}(2 \mathrm{~s}, 2 \mathrm{p})$, which determine the energies of certain states centered at the average energy of a configuration with a multiplet. These six parameters were determined by a separate search of $3^{6}=729$ combinations per cycle. This faster cycle was repeated and optimized for a given step size before switching to the 9-parameter cycle. The six parameters could be optimized separately because they do not change the value of the average energies of the configuration, but change the spacing among states. Computations were made in double precision on a personal computer with a $\sim 2000$-line FORTRAN program.

\section{Results}

The sum-squared error between 587 experimental and calculated energies in this work was 0.58 (a.u.) $)^{2}$. The average deviation was \pm 0.023 a.u. and the standard deviation with the experimental energies with 15 degrees of freedom was 0.033 a.u. In the present work, $98 \%$ of the calculated energy levels have a percent relative error within $\pm 0.1 \%$ of the corresponding experimental value. Also, $49 \%$ of the energies have a percent relative error less than $\pm 0.01 \%$ of the experimental energy. The 587 energy levels correspond to 293 different electron configurations.

Three experimental energies were rejected because they each had a deviation greater than 0.8 a.u. and two more experimental energies were rejected by the criterion of having a deviation greater than 0.16 a.u. with the calculated energy. Chauvenet's criterion for rejecting data within a population of $\sim 600$ points is at 3.3-sigma deviation, which corresponds to deviations greater than 0.11 a.u.

The numerical values of the parameters determined by least-squares analysis are shown in Equations (12) through (17). The $G^{1}$ and $F^{2}$ values have units of a.u.

$$
\begin{gathered}
Z_{1 S}=Z-\left[0.3125-0.030 / Z-0.006 / Z^{2}\right]\left(N_{1 S}-1\right)-0.0221 N_{2 S}-0.0074 N_{2 P} \\
Z_{2 S}=Z-0.6989 N_{1 S}-0.3008\left(N_{2 S}-1\right)-0.2299 N_{2 P} \\
Z_{2 P}=Z-0.9270 N_{1 S}-0.3668 N_{2 S}-[0.3526-0.1618 / Z]\left(N_{2 P}-1\right) \\
G^{1}(1 \mathrm{~s}, 2 \mathrm{p})=0.039 Z_{2 P}-0.028
\end{gathered}
$$




$$
\begin{gathered}
F^{2}(2 \mathrm{p}, 2 \mathrm{p})=\left(0.092-0.009 N_{2 S}\right) Z_{2 P} \\
G^{1}(2 \mathrm{~s}, 2 \mathrm{p})=0.092 Z_{2 P}
\end{gathered}
$$

The values of the screening parameters given in Equations (12) through (14) are similar to those of other researchers [5] [7]. In Equation (12), the expression multiplying $\left(N_{1 S}-1\right)$ was obtained in this work by fitting three terms to the highly accurate solutions of the Schrödinger equation for 2-electron energies from Nakashima [25] where $E\left(1 \mathrm{~s}^{2}\right)$ equals $-2 Z_{1 S}^{2} / 2$, which gave $Z_{1 S}=Z-\left(0.3125-0.30 / Z-0.006 / Z^{2}\right)$, for $Z$ equal to 2 through 10 . (The energies of that work did not include $E_{R E L}$.) The value 0.3125 is a theoretical value [5] [7] that can be calculated for the screening between two 1s electrons. The terms that are divided by $Z$ and $Z^{2}$ correct for the $Z$-dependence of the screening. The factors, -0.0221 and -0.0074 , in Equation (12), which account for 1s screening by $2 \mathrm{~s}$ and $2 \mathrm{p}$ electrons, are significant to the computations. The screening parameter between $2 s$ electrons has a theoretical value [5] [7] of 0.3008 , which matches the value shown in Equation (13). Layzer [5] calculated the screening parameter between $2 p$ electrons to be 0.3492 . The parameter shown in Equation (14) is 0.3526 with the term $-0.1616 / Z$ to correct for a $Z$ dependence of the screening of $Z_{2 P}$ by $2 \mathrm{p}$ electrons.

Estimates of $G^{1}(1 \mathrm{~s}, 2 \mathrm{p}), F^{2}(2 \mathrm{p}, 2 \mathrm{p})$ and $G^{1}(2 \mathrm{~s}, 2 \mathrm{p})$ from Equations (15) through (17) have enabled the calculation of energies to proceed smoothly. $G^{1}$ (1s, 2p) has been calculated by Golden [26] to be $0.0512 Z_{2 \mathrm{P}}$ and is shown in Equation (15) to be $0.039 Z_{2 P}-0.028$ where the negative term has been introduced empirically in this work. Both $F^{2}(2 \mathrm{p}, 2 \mathrm{p})$ and $G^{1}(2 \mathrm{~s}, 2 \mathrm{p})$ have been calculated with Slater wave functions by Golden [26] to be $0.0879 Z_{2 P} . F^{2}(2 \mathrm{p}, 2 \mathrm{p})$ is shown in Equation (16) to equal $0.092 Z_{2 P}$, or $0.083 Z_{2 P}$, or $0.074 Z_{2 P}$ for configurations in which $N_{2 S}$ is 0 , 1, or 2, respectively; whereas, $G^{1}(2 \mathrm{~s}, 2 \mathrm{p})$ is shown in Equation (17) to be $0.092 Z_{2 P}$.

Experimental values of $G^{1}(1 \mathrm{~s}, 2 \mathrm{p}), F^{2}(2 \mathrm{p}, 2 \mathrm{p})$ and $G^{1}(2 \mathrm{~s}, 2 \mathrm{p})$ were determined by a least-squares fit of the experimental energies of the corresponding states listed in Table 1. Slater (see [2]: p. 344) stated that the experimental data deviated from the equations due to configuration interaction that sometimes lowered the least-negative state of a given configuration. Figures 1-3 show the comparisons between $G^{1}(1 \mathrm{~s}, 2 \mathrm{p}), F^{2}(2 \mathrm{p}, 2 \mathrm{p})$ and $G^{1}(2 \mathrm{~s}, 2 \mathrm{p})$ values calculated from experimental energies with the equations shown in Table 1 and calculated with Equations (15) through (17), respectively. The twelve red points in Figure 2, which have a different slope, correspond to configurations $1 s^{2} 2 s^{0} 2 p^{2}$ and $1 s^{2} 2 s^{0} 2 p^{4}$.

The relativistic energies, $E_{R E L}$, were easily calculated for all electron configurations by Equation (5). $E_{R E L}$ for configurations with two, four or ten electrons in closed subshells are compared in Table 2 to values derived from perturbation methods by Hartmann ([21]: Table IV) who used Hartree-Fock functions for atoms and ions up to $Z=36$ and ions with up to eighteen electrons. The Retherford-Lamb correction for 1 s electrons and the Breit interaction were included by 


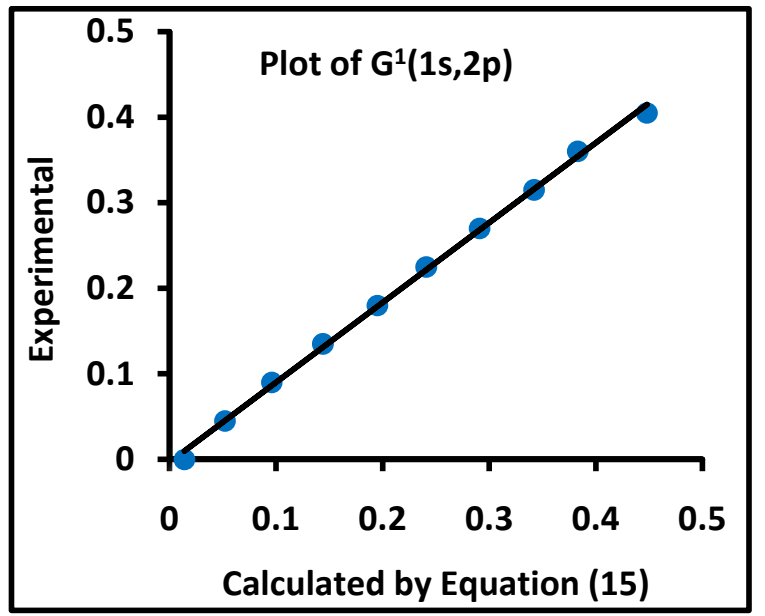

Figure 1. Plot of experimental versus calculated values of $G^{1}(1 \mathrm{~s}, 2 \mathrm{p})$ in a.u.

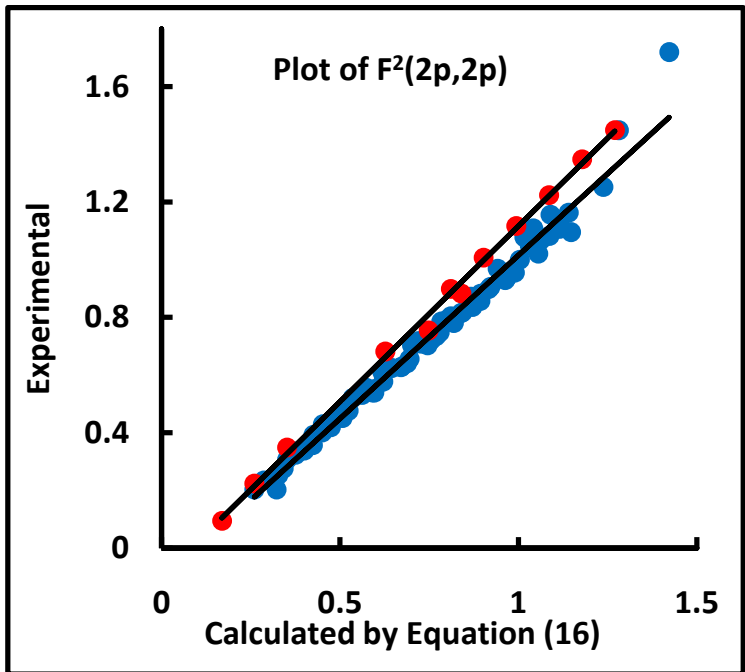

Figure 2. Plot of experimental versus calculated values of $F^{2}(2 \mathrm{p}, 2 \mathrm{p})$ in a.u. The red points correspond to twelve values for configurations $1 \mathrm{~s}^{2} 2 \mathrm{~s}^{0} 2 \mathrm{p}^{2}$ and $1 \mathrm{~s}^{2} 2 \mathrm{~s}^{0} 2 \mathrm{p}^{4}$.

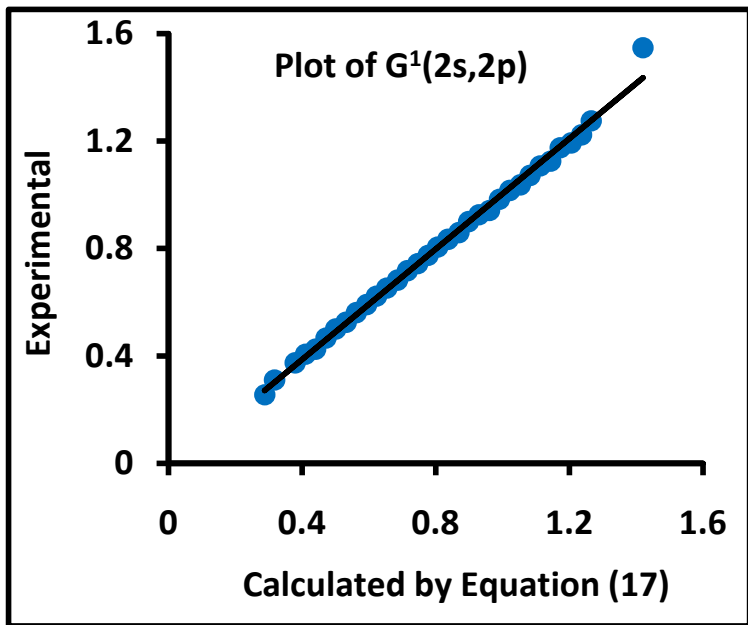

Figure 3. Plot of Experimental versus calculated values of $G^{1}(2 \mathrm{~s}, 2 \mathrm{p})$ in a.u. 
Table 2. $E_{R E L}$ (in a.u.), calculated by Equation (5) for ${ }^{1} \mathrm{~S}_{\mathrm{o}}$ states of 2-, 4-, and 10-electron atoms/ions, compared to the corresponding values of Hartmann [21].

\begin{tabular}{|c|c|c|c|c|c|c|c|c|c|}
\hline \multicolumn{4}{|c|}{2 Electrons } & \multicolumn{3}{|c|}{4 Electrons } & \multicolumn{3}{|c|}{10 Electrons } \\
\hline$Z$ & Eqn (5) & $\operatorname{Ref}[21]$ & Diff & Eqn (5) & $\operatorname{Ref}[21]$ & Diff & Eqn (5) & $\operatorname{Ref}[21]$ & Diff \\
\hline 2 & -0.00009 & -0.00007 & --- & & & & & & \\
\hline 3 & -0.00060 & -0.00054 & --- & & & & & & \\
\hline 4 & -0.0022 & -0.0021 & $-5 \%$ & -0.0022 & -0.0022 & $0 \%$ & & & \\
\hline 5 & -0.0057 & -0.0057 & $0 \%$ & -0.0060 & -0.0062 & $3 \%$ & & & \\
\hline 6 & -0.0125 & -0.0125 & $0 \%$ & -0.0135 & -0.0141 & $4 \%$ & & & \\
\hline 7 & -0.0242 & -0.0243 & $0 \%$ & -0.0268 & -0.0280 & $4 \%$ & & & \\
\hline 8 & -0.0426 & -0.0429 & $1 \%$ & -0.0481 & -0.0503 & $4 \%$ & & & \\
\hline 9 & -0.0699 & -0.0706 & $1 \%$ & -0.0802 & -0.0839 & $4 \%$ & & & \\
\hline 10 & -0.1088 & -0.1099 & $1 \%$ & -0.1264 & -0.1320 & $4 \%$ & -0.1276 & -0.1313 & $3 \%$ \\
\hline 11 & -0.1620 & -0.1637 & $1 \%$ & -0.1903 & -0.1983 & $4 \%$ & -0.1982 & -0.2007 & $1 \%$ \\
\hline 12 & -0.2328 & -0.2352 & $1 \%$ & -0.2759 & -0.2869 & $4 \%$ & -0.2960 & -0.2951 & $0 \%$ \\
\hline 13 & -0.3248 & -0.3279 & $1 \%$ & -0.3878 & -0.4022 & $4 \%$ & -0.4274 & -0.4200 & $-2 \%$ \\
\hline 14 & -0.4417 & -0.4457 & $1 \%$ & -0.5309 & -0.5493 & $3 \%$ & -0.5997 & -0.5815 & $-3 \%$ \\
\hline 15 & -0.5878 & -0.5927 & $1 \%$ & -0.7106 & -0.7334 & $3 \%$ & -0.8208 & -0.7862 & $-4 \%$ \\
\hline 16 & -0.7676 & -0.7735 & $1 \%$ & -0.9326 & -0.9603 & $3 \%$ & -1.0994 & -1.0413 & $-6 \%$ \\
\hline 17 & -0.9860 & -0.9928 & $1 \%$ & -1.2032 & -1.2363 & $3 \%$ & -1.4450 & -1.3544 & $-7 \%$ \\
\hline 18 & -1.2483 & -1.2557 & $1 \%$ & -1.5291 & -1.5679 & $2 \%$ & -1.8676 & -1.7339 & $-8 \%$ \\
\hline
\end{tabular}

Hartmann [21]. In the present work, the Lamb correction was included in Equation (5) for the 1s electrons, but the Breit interaction was not included. The agreement between the sets of values for $E_{R E L}$ shown in Table 2 is approximately $\pm 5 \%$. Similar values of $E_{R E L}$ for systems with two, four or ten electrons are given by Veillard [24] and Anno ([27]: Tables II and III; [28]).

Equation (4) for $E_{R E L}$ can be compared to Equation (5). The closed subshell systems shown in Table 2 with two, four or ten electrons each have the term ${ }^{1} \mathrm{~S}_{0}$, where the subscript zero is the value of $j$. If Equation (4) had been used for configurations containing two 1 s electrons, then the factor $\left[1 /(j+1 / 2)-3 /\left(4 n_{i}\right)\right]$ would equal $5 / 4$ for $n_{i}=1$ with $j=0$, which is a five-times higher factor than $\left[1-3 /\left(4 n_{i}\right)\right]=1 / 4$ of Equation (5). The smaller term $\Lambda_{i, Z}$ of Equation (5) has been ignored in this comparison. For $2 s$ and $2 p$ electrons, the factor

$\left[1 /(j+1 / 2)-3 /\left(4 n_{i}\right)\right]$ would equal $13 / 8$ for $n_{i}=2$ with $j=0$, which is 2.6 -times larger than $\left[1-3 /\left(4 n_{i}\right)\right]=5 / 8$ of Equation (5). Therefore, if Equation (4) had been used in the present work, the values of $E_{R E L}$ for the 2-electron system would have increased in magnitude by a factor of five and the values of the 4and 10-electron systems, which have a major contribution from the 1s electrons, would have increased in magnitude by a factor of $\sim 4$ or more compared to the values of $E_{R E L}$ shown in Table 2. 
$E_{R E L}$ was also calculated for 2, 4, and 10 electrons for values of $Z$ from 19 to 36 with Equation (5) and Equations (12) through (14) and with the Lamb corrections for two 1s electrons, as previously described. These values were compared to the corresponding values of $E_{R E L}$ given by Hartmann ([21]: Table IV). The values of $E_{R E L}$ for 2-electron and 4-electron atoms and ions for values of $Z$ up to 36 agreed within $\sim 1 \%$. The values of $E_{R E L}$ for the 10-electron atoms and ions for values of $Z$ up to 36 were more negative by $\sim 8 \%$ at a $Z$ of 18 and by $\sim 20 \%$ for a $Z$ of 36. Since value of $E_{R E L}$ of the 10-electron atom and ions involved the addition of six 2p electrons, an empirical change of $j=1 / 2$ to $j=\sim(1 / 2+\ell / 4)$, where $\ell=0$ for $\mathrm{s}$ orbitals and $\ell=1$ for $\mathrm{p}$ orbitals, might improve the agreement of the calculation of $E_{R E L}$ to that of Hartmann [21], especially at higher values of $Z$.

Good overall agreement between the calculated and observed energies was obtained with a constant value of $j=1 / 2$ used in Equation (5). Also, the Dirac relationship, shown in Equation (1), gave similar agreement between the 587 calculated and observed energies when it was modified to sum electron energies with a constant value of $j=1 / 2$, with $Z_{i}$ substituted for $Z$, and with $-\Lambda_{i, Z}$ (for the number of $1 \mathrm{~s}$ electrons that were present). After the parameters were allowed to vary, the calculation with the modified Equation (1) gave similar parameters to those of Equation (3) with $E_{R E L}$ calculated by Equation (5). The sum-squared error was 0.60 , compared to 0.58 (a.u. $)^{2}$ for the agreement with Equation (3).

When $j$ was set to $S+L$, the agreement between calculated and experimental electron energies was poor. However, when $j$ was set to $1 / 2$, the overall agreement was favorable, the calculations were simplified for all of the multi-electron configurations and the values of $E_{R E L}$ agreed well with those given in Table 2 and with additional values of Hartmann [21] for values of $E_{R E L}$ for $Z$ up to 36 .

\section{Hydrogen-Like Wave Functions Associated with $Z_{1 S}, Z_{2 S}$ and $Z_{2 P}$}

The individual energies $E_{i}$ of the $1 \mathrm{~s}, 2 \mathrm{~s}$ and $2 \mathrm{p}$ electrons within multi-electron configurations equal $-Z_{i}^{2} /\left(2 n_{i}^{2}\right)$ plus $E_{R E L}$ in a.u., where $Z_{1 S}, Z_{2 S}$ and $Z_{2 P}$ are the effective nuclear charges of hydrogen-like wave functions. (Slater $1 \mathrm{~s}$ and $2 \mathrm{p}$ wave functions each correspond to hydrogen-like $1 \mathrm{~s}$ and $2 \mathrm{p}$ wave functions, but the node-less Slater $2 s$ wave function does not correspond to the hydrogen-like 2s wave function.) When $Z_{1 S}$ and $Z_{2 S}$ are unequal, the 1s and 2s hydrogen-like wave functions are not orthogonal. However, the modified wave function $\Phi_{2 S, \text { SCHMIDT }}$ has been made orthogonal to $\Phi_{1 S}$ by the Gray-Schmidt method:

$$
\Phi_{2 S, \text { SCHмIDT }}=\left(\Phi_{2 S}-S \Phi_{1 S}\right) /\left(1-S^{2}\right)^{1 / 2}
$$

where $\Phi_{1 S}$ and $\Phi_{2 S}$ are normalized hydrogen-like wave functions. The overlap integral $S$ between $\Phi_{1 S}$ and $\Phi_{2 S}$ with $Z_{1 S} \neq Z_{2 S}$ equals the following:

$$
S=\int \Phi_{1 S} \Phi_{2 S} \partial \tau=8^{1 / 2} Z_{1 S}^{3 / 2} Z_{2 S}^{3 / 2}\left(Z_{1 S}-Z_{2 S}\right) /\left(Z_{1 S}+0.5 Z_{2 S}\right)^{4} .
$$

One can substitute $\Phi_{2 S, \text { SCHMIDT }}$ from Equation (18) into the expression below, 
where $\hat{H} \Phi_{1 S}=E_{1 S} \Phi_{1 S}$ and $\hat{H} \Phi_{2 S}=E_{2 S} \Phi_{2 S}$, to demonstrate that $E_{2 S, S C H M I D T}$ equals $E_{2 s}$.

$$
E_{2 S, \text { SCHMIDT }}=\int \Phi_{2 S, \text { SCHMIDT }} \hat{H} \Phi_{2 S, \text { SCHMIDT }} \partial \tau=E_{2 S}
$$

In addition to enabling the calculation of the energies of numerous configurations of light atoms and ions with up to 10 electrons for $Z$ up to 18 , the values of $Z_{1 S}, Z_{2 S}$ and $Z_{2 P}$, given in Equations (12) through (14), can be used to form wave functions for future calculations. The energy of a given hydrogen-like wave function corresponds closely to the average experimental energy of its electron configuration.

\section{Conclusions}

The Screened Hydrogenic Model (SHM) has been used to calculate energies that closely match the experimental energies of light atoms and ions with $1 \mathrm{~s}, 2 \mathrm{~s}$ and 2 p electrons for up to 10 total electrons and for atomic numbers up to 18 . The total experimental energies for the atoms with $Z=1$ through 10 each agreed within \pm 0.001 a.u. to those tabulated by Veillard [24], except for oxygen $(Z=8)$ that agreed within \pm 0.02 a.u. (compared to its total energy of -75.1101 a.u.).

The standard deviation for the 587 energies was 0.033 a.u. Also, $98 \%$ of the calculated energies had a percent error of $\pm 0.1 \%$ relative to the corresponding experimental energy and $49 \%$ of the calculated energies had a percent error of $\pm 0.01 \%$ relative to the corresponding experimental energies. Only five data points were rejected from the original set of experimental energies. Nine parameters were used to account for the screening of electrons among the electrons in the $1 \mathrm{~s}$, $2 s$, and $2 p$ orbitals. Multiplets due to Russell-Saunders coupling were calculated with $G^{1}(1 \mathrm{~s}, 2 \mathrm{~s}), F^{2}(2 \mathrm{p}, 2 \mathrm{p})$ and $G^{1}(2 \mathrm{~s}, 2 \mathrm{p})$ obtained with six additional parameters.

Equation (5) with a constant value of $j=1 / 2$ has been proposed for the calculation of $E_{R E L}$ for all multi-electron configurations in order to achieve favorable overall agreement. As shown in Table 2, the values of $E_{R E L}$ calculated with Equation (5) for configurations with two, four, and ten electrons agree well with those calculated by past researchers.

In the future, these calculations could be expanded to include electrons in higher shells with additional screening parameters. The Retherford-Lamb corrections could be added for more electrons. The Breit interaction and other refinements might also be included.

\section{Acknowledgements}

The author is an employee of Consolidated Nuclear Security, LLC (CNS), a subcontractor of an agency of the United States Government under Contract DE-NA-0001942.

\section{Conflicts of Interest}

The author declares no conflicts of interest regarding the publication of this paper. 


\section{References}

[1] Slater, J.C. (1930) Atomic Shielding Constants. Physical Review Journals, 36, 57-64. https://doi.org/10.1103/PhysRev.36.57

[2] Slater, J.C. (1960) Quantum Theory of Atomic Structure. Vol. 1, McGraw-Hill Book Company, New York.

[3] Layzer, D. (1959) On a Screening Theory of Atomic Spectra. Annals of Physics, 8, 271-296. https://doi.org/10.1016/0003-4916(59)90023-5

[4] Layzer, D. and Bahcall, J. (1962) Relativistic Z-Dependent Theory of Many-Electron Atoms. Annals of Physics, 17, 177-204. https://doi.org/10.1016/0003-4916(62)90024-6

[5] Layzer, D. (1967) Z-Expansion Calculations of Energy Levels and Transition Probabilities in Many-Electron Atoms. International Journal of Quantum Chemistry, 1, 45-59. https://doi.org/10.1002/qua.560010605

[6] Kregar, M. (1985) The Virial as the Atomic Model Potential Energy Operator. Physica Scripta, 31, 246-254. https://doi.org/10.1088/0031-8949/31/4/005

[7] Kregar, M. (1986) On the Description of Many-Electron Atoms. Il Nuovo Cimento $D, 8,159-176$. https://doi.org/10.1007/BF02450286

[8] More, R.M. (1982) Electronic Energy-Levels in Dense Plasmas. Journal of Quantitative Spectroscopy and Radiative Transfer, 27, 345-357. https://doi.org/10.1016/0022-4073(82)90127-3

[9] Faussurier, G., Blancard, C. and Decoster, A. (1997) New Screening Coefficients for the Hydrogenic Ion Model Including L-Splitting for Fast Calculations of Atomic Structure in Plasmas. Journal of Quantitative Spectroscopy and Radiative Transfer, 58, 233-260. https://doi.org/10.1016/S0022-4073(97)00018-6

[10] Di Rocco, H.O. (2002) A Simple and Effective Approach to Calculate the Energy of Complex Atoms. Revista Mexicana de Física, 48, 76-87.

[11] Rubiano, J.G., Rodriguez, R., Gil, J.M., Ruano, F.H., Martel, P. and Minguez, E. (2002) A Screened Hydrogenic Model Using Analytical Potentials. Journal of Quantitative Spectroscopy and Radiative Transfer, 72, 575-588. https://doi.org/10.1016/S0022-4073(01)00142-X

[12] Smith, C.C. (2011) A Screened Hydrogenic Model with Fine Structure Splitting. High Energy Density Physics, 7, 1-5. https://doi.org/10.1016/j.hedp.2010.11.001

[13] Mendoza, M.A., Rubiano, J.G., Gil, J.M., Rodriguez, R., Florido, R., Martel, P. and Minguez, E. (2011) A New Set of Relativistic Screening Constants for the Screened Hydrogenic Model. High Energy Density Phys, 7, 169-179.

https://doi.org/10.1016/j.hedp.2011.04.006

[14] Lanzini, F. and Di Rocco, H.O. (2015) Screening Parameters for the Relativistic Hydrogenic Model. High Energy Density Phys, 17, 240-247.

https://doi.org/10.1016/j.hedp.2015.08.002

[15] Bethe, H.E. and Salpeter, E.E (2014) Quantum Mechanics of One- and Two-Electron Atoms. Martino Publishing, Mansfield Centre, Connecticut.

[16] Mitsushima, M. (1970) Quantum Mechanics of Atomic Spectra and Atomic Structure. W.A. Benjamin, Inc., New York.

[17] Snyder, R. (1971) A Formula for Relativistic Atomic Energies. Journal of Physics B: Atomic and Molecular Physics, 4, 1150-1162. https://doi.org/10.1088/0022-3700/4/9/004

[18] Snyder, R. (1972) A Formula for Relativistic Atomic Energies: II. Journal of Physics 
B: Atomic and Molecular Physics, 5, 934-942. https://doi.org/10.1088/0022-3700/5/5/014

[19] Kabir, P.K. and Salpeter, E.E. (1957) Radiative Corrections to the Ground-State Energy of the Helium Atom. Physical Review Journals, 108, 1256-1263.

https://doi.org/10.1103/PhysRev.108.1256

[20] Garcia, J.D. and Mack, J.E. (1965) Energy Levels and Line Tables for One-Electron Atomic Spectra. Journal of the Optical Society of America, 55, 654-685. https://doi.org/10.1364/JOSA.55.000654

[21] Hartmann, H. and Clementi E. (1964) Relativistic Correction for Analytic HartreeFock Wave Functions. Physical Review Journals, 133, A1295-A1299. https://doi.org/10.1103/PhysRev.133.A1295

[22] Bashkin, S. and Stoner Jr., J. (1975) Atomic Energy Levels and Grotrian Diagrams (Hydrogen I-Phosphorus XV). Vol. 1, North-Holland Publishing Co., Amsterdam. https://doi.org/10.1016/C2013-0-04477-5

[23] Bashkin, S. and Stoner Jr., J. (1978) Atomic Energy Levels and Grotrian Diagrams (Sulfur-Titanium). Vol. 2, North-Holland Publishing Co., Amsterdam.

[24] Veillard, A. and Clementi, E. (1968) Correlation Energy in Atomic Systems. V. Degeneracy Effects for the Second-Row Atoms. The Journal of Chemical Physics, 49, 2415-2421. https://doi.org/10.1063/1.1670415

[25] Nakashima, H. and Nakatsuji, H. (2007) Solving the Schrödinger Equation for Helium Atom and Its Isoelectronic Ions with the Free Iterative Complement Interaction (ICI) Method. The Journal of Chemical Physics, 127, Article ID: 224104. https://doi.org/10.1063/1.2801981

[26] Golden, L.B. (1978) Exact Slater Integrals. Computer Physics Communications, 14, 255-260. https://doi.org/10.1016/0010-4655(78)90018-8

[27] Anno, T. and Teruya, H. (1989) Relativistic Effect on Total Energies for the Determination of Correlation Energies of Atoms from their Experimental Total Energies. The Journal of Chemical Physics, 91, 4738-4744. https://doi.org/10.1063/1.456763

[28] Anno, T. and Teruya, H. (1992) Erratum: Relativistic Effect on Total Energies for the Determination of Correlation Energies of Atoms from their Experimental Total Energies. The Journal of Chemical Physics, 97, Article No. 2174.

https://doi.org/10.1063/1.463993 